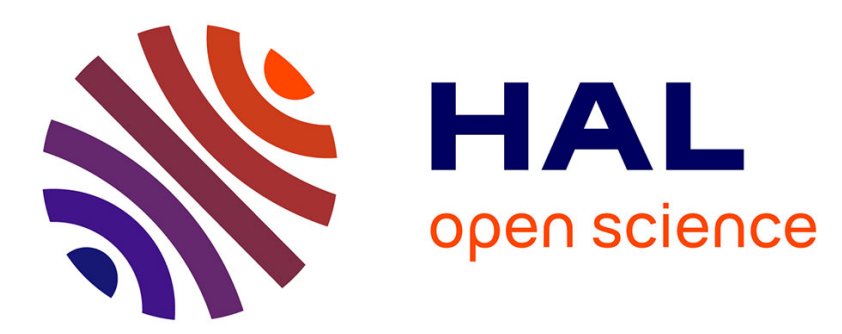

\title{
Shape and topology sensitivity analysis for cracks in elastic bodies on boundaries of rigid inclusions
}

Alexander Khludnev, Andre Novotny, Jan Sokolowski, Antoni Zochowski

\section{To cite this version:}

Alexander Khludnev, Andre Novotny, Jan Sokolowski, Antoni Zochowski. Shape and topology sensitivity analysis for cracks in elastic bodies on boundaries of rigid inclusions. 2008. hal-00335565

\section{HAL Id: hal-00335565 https://hal.science/hal-00335565}

Preprint submitted on 29 Oct 2008

HAL is a multi-disciplinary open access archive for the deposit and dissemination of scientific research documents, whether they are published or not. The documents may come from teaching and research institutions in France or abroad, or from public or private research centers.
L'archive ouverte pluridisciplinaire HAL, est destinée au dépôt et à la diffusion de documents scientifiques de niveau recherche, publiés ou non, émanant des établissements d'enseignement et de recherche français ou étrangers, des laboratoires publics ou privés. 


\title{
Shape and topology sensitivity analysis for cracks in elastic bodies on boundaries of rigid inclusions
}

\author{
A.M. Khludnev ${ }^{\text {a }}$ A.A. Novotny ${ }^{\mathrm{b}}$ J. Sokołowski ${ }^{\mathrm{c}, *}$ \\ A. Żochowski ${ }^{\mathrm{d}}$ \\ ${ }^{a}$ Lavrentyev Institute of Hydrodynamics. Novosibirsk, Russia. \\ ${ }^{\mathrm{b}}$ Laboratório Nacional de Computação Científica LNCC/MCT \\ Av. Getúlio Vargas 333, 25651-075 Petrópolis - RJ, Brasil. \\ ${ }^{\mathrm{c}}$ Institut Elie Cartan, UMR 7502 (Nancy Université, CNRS, INRIA), \\ Laboratoire de Mathématiques, Université Henri Poincaré Nancy I, \\ B.P. 239, 54506 Vandoeuvre-lès-Nancy Cedex, France. \\ ${ }^{\mathrm{d}}$ Systems Research Institute of the Polish Academy of Sciences.
}

\begin{abstract}
We consider a 3D elastic body with a rigid inclusion and a crack located at the boundary of the inclusion. It is assumed that non-penetration conditions are imposed at the crack faces which do not allow the opposite crack faces to penetrate each other. We analyze the variational formulation of the problem and provide shape and topology sensitivity analysis of the solution.
\end{abstract}

Key words: Rigid inclusion, crack growth, contact condition, shape sensitivity analysis, topological derivatives.

\section{Introduction}

The inclusions in elastic bodies are also important for applications, both in design procedures and in numerical solution of some inverse problems. We restrict ourselves to the limit case of a rigid inclusion, with a crack at the interface. This seems to be a new class of problems, both for the analysis and

\footnotetext{
* Corresponding author.

Email address: Jan.Sokolowski@iecn.u-nancy.fr (J. Sokołowski).
} 


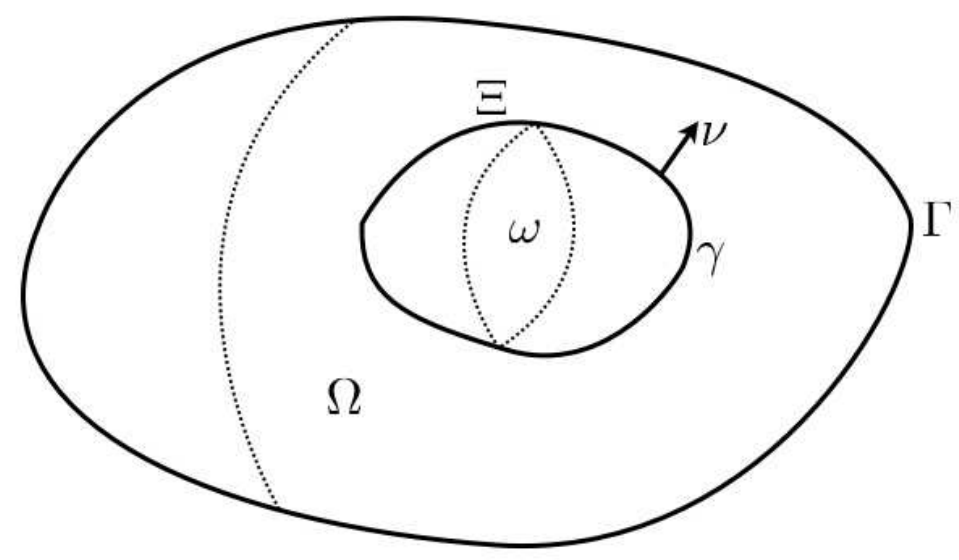

Fig. 1. Domain $\Omega$ with rigid inclusion $\omega$

for the shape optimization. One can also attempt to find the shape derivative of the elastic energy with respect to the perturbations of the crack tip, some results in this direction are given with all details in section 5 .

\section{Problem formulation}

Let $\Omega \subset \mathbb{R}^{3}$ be a bounded domain with smooth boundary $\Gamma$, and $\omega \subset \Omega$ be a subdomain with smooth boundary $\Xi$ such that $\bar{\omega} \cap \Gamma=\emptyset$. We assume that $\Xi$ consists of two parts $\gamma$ and $\Xi \backslash \gamma$, meas $(\Xi \backslash \gamma)>0$, where $\gamma$ is a smooth 2D surface described as

$$
x_{i}=x_{i}\left(y_{1}, y_{2}\right), \quad\left(y_{1}, y_{2}\right) \in D, i=1,2,3 \text {, }
$$

with bounded domain $D \subset \mathbb{R}^{2}$ having a smooth boundary $\partial D$, and a rank of the matrix $\frac{\partial x}{\partial y}$ is equal to 2 .

Denote by $\nu=\left(\nu_{1}, \nu_{2}, \nu_{3}\right)$ a unit outward normal vector to $\Xi$, see Fig. 1 . The subdomain $\omega$ is assumed to correspond to a rigid inclusion, and the surface $\gamma$ describes a crack located on $\Xi$. Domain $\Omega \backslash \bar{\omega}$ corresponds to the elastic part of the body. For the further use we introduce the space of infinitesimal rigid displacements

$$
R(\omega)=\left\{\rho=\left(\rho_{1}, \rho_{2}, \rho_{3}\right) \mid \rho(x)=B x+C, x \in \omega\right\}
$$


where

$$
B=\left(\begin{array}{ccc}
0 & b_{12} & b_{13} \\
-b_{12} & 0 & b_{23} \\
-b_{13} & -b_{23} & 0
\end{array}\right), \quad C=\left(c^{1}, c^{2}, c^{3}\right) ; \quad b_{i j}, c^{i}=\text { const }, i, j=1,2,3 .
$$

Denote $\Omega_{\gamma}=\Omega \backslash \bar{\gamma}$. Problem formulation describing an equilibrium of the elastic body with the rigid inclusion $\omega$ and the crack $\gamma$ is as follows. In the domain $\Omega_{\gamma}$, we have to find functions $u=\left(u_{1}, u_{2}, u_{3}\right), u=\rho_{0}$ on $\omega ; \rho_{0} \in R(\omega)$; and in the domain $\Omega \backslash \bar{\omega}$ we have to find functions $\sigma=\left\{\sigma_{i j}\right\}, i, j=1,2,3$, such that

$$
\begin{aligned}
-\operatorname{div} \sigma=F & \text { in } \quad \Omega \backslash \bar{\omega} \\
\sigma-A \varepsilon(u)=0 & \text { in } \Omega \backslash \bar{\omega}, \\
u=0 & \text { on } \Gamma, \\
\left(u-\rho_{0}\right) \cdot \nu \geq 0 & \text { on } \gamma^{+}, \\
\sigma_{\tau}=0, \sigma_{\nu} \leq 0 & \text { on } \gamma^{+}, \\
\sigma_{\nu}\left(u-\rho_{0}\right) \cdot \nu=0 & \text { on } \gamma^{+}, \\
-\int_{\Xi} \sigma \nu \cdot \rho=\int_{\omega} F \cdot \rho & \forall \rho \in R(\omega) .
\end{aligned}
$$

Here $F=\left(F_{1}, F_{2}, F_{3}\right) \in L^{2}(\Omega)$ is a given function,

$$
\begin{aligned}
\sigma_{\nu} & =\sigma_{i j} \nu_{j} \nu_{i}, \quad \sigma_{\tau}=\sigma \nu-\sigma_{\nu} \nu, \\
\sigma_{\tau} & =\left(\sigma_{\tau}^{1}, \sigma_{\tau}^{2}, \sigma_{\tau}^{3}\right), \quad \sigma \nu=\left\{\sigma_{i j} \nu_{j}\right\}_{i=1}^{i=3}, \\
\varepsilon_{i j}(u) & =\frac{1}{2}\left(u_{i, j}+u_{j, i}\right), \quad i, j=1,2,3 .
\end{aligned}
$$

All functions with two below indices are assumed to be symmetric in those indices. Summation convention over repeated indices is accepted throughout the paper. Elasticity tensor $A=\left\{a_{i j k l}\right\}, i, j, k, l=1,2,3$, is given, and it satisfies a symmetry and positive definiteness properties,

$$
\begin{gathered}
a_{i j k l}=a_{k l i j}=a_{j i k l}, \quad a_{i j k l} \in L^{\infty}(\Omega), i, j, k, l=1,2,3, \\
a_{i j k l} \xi_{k l} \xi_{i j} \geq c_{0}|\xi|^{2}, \quad \forall \xi_{i j}=\xi_{j i}, \quad c_{0}=\text { const. }
\end{gathered}
$$

In addition, we consider the isotropic case, namely

$$
A=2 m \mathbb{I}+l(\mathrm{I} \otimes \mathrm{I}),
$$

where I and $\mathbb{I}$ respectively are the second and fourth order identity tensors and, $m$ and $l$ are the Lamé coefficients, which can be defined in terms of the 
Young modulus $E$ and the Poisson ratio $v$ as

$$
m=\frac{E}{2(1+v)} \quad \text { and } \quad l=\frac{v E}{(1+v)(1-2 v)} .
$$

Relations (1) are equilibrium equations, and (2) corresponds to the Hooke's law. Inequality (4) describes a mutual nonpenetration between crack faces $\gamma^{ \pm}$. The first relation in (5) means a zero friction between the crack faces. For simplicity we assume a clamping condition (3) on $\Gamma$.

Note that external forces $F$ are applied to $\Omega \backslash \bar{\omega}$ as well as to $\omega$, but there are no equilibrium equations in $\omega$. Influence of these forces is taken into account through (7). If we have no crack $\gamma$ on $\Xi$, relations (4)-(6) should be omitted. This particular problem formulation for the particular case $F=0$ in $\omega$ can be found in [22].

First of all we provide a variational formulation of the problem (1)-(7). To this end, introduce the Sobolev space

$$
H_{\Gamma}^{1, \omega}\left(\Omega_{\gamma}\right)=\left\{v \in H^{1}\left(\Omega_{\gamma}\right)^{3} \mid \varepsilon(v)=0 \text { on } \omega ; v=0 \text { on } \Gamma\right\}
$$

and define the set of admissible displacements

$$
K_{\omega}=\left\{v \in H_{\Gamma}^{1, \omega}\left(\Omega_{\gamma}\right) \mid \varepsilon(v)=0 \text { on } \omega ;\left(v^{+}-v^{-}\right) \cdot \nu \geq 0 \text { on } \gamma\right\} .
$$

Let $(\cdot, \cdot)_{\Omega \backslash \bar{\omega}}$ be the inner product in $L^{2}(\Omega \backslash \bar{\omega})$. Consider the energy functional

$$
\Pi(v)=\frac{1}{2}(\sigma(v), \varepsilon(v))_{\Omega \backslash \bar{\omega}}-(F, v)_{\Omega_{\gamma}},
$$

where $\sigma(v)=\sigma$ are defined from (2) for $u=v$, and a minimization problem

$$
\inf _{v \in K_{\omega}} \Pi(v) .
$$

The set $K_{\omega}$ is weakly closed in the space $H_{\Gamma}^{1, \omega}\left(\Omega_{\gamma}\right)$, and the functional $\Pi$ is coercive and weakly lower semicontinuous on this space. Hence the problem (13) has a solution satisfying the variational inequality

$$
\begin{aligned}
u & \in K_{\omega}, \\
(\sigma(u), \varepsilon(\bar{u}-u))_{\Omega \backslash \bar{\omega}} & \geq(F, \bar{u}-u)_{\Omega_{\gamma}} \quad \forall \bar{u} \in K_{\omega} .
\end{aligned}
$$

Solution $u$ of the problem (14)-(15) is unique.

Assuming that the solution of (14)-(15) is quite smooth we can derive all relations (1)-(7), and conversely, any smooth solution of (1)-(7) satisfies (14)(15). On the other hand, the solution smoothness of (14)-(15) does not provide 
a fulfillment of (5)-(6) in a point-wise sense, and this point requires some explanations. To this end, introduce the weighted Sobolev space

$$
H_{00}^{1 / 2}(\gamma)=\left\{v \in H^{1 / 2}(\gamma) \mid \int_{\gamma} \frac{v^{2}}{r}<+\infty\right\}
$$

where $r(x)=\operatorname{dist}(x, \partial \gamma)$, and denote by $H_{00}^{-1 / 2}(\gamma)$ a space dual of $H_{00}^{1 / 2}$. Since $\sigma, \operatorname{div} \sigma \in L^{2}(\Omega \backslash \bar{\omega})$ we have (see $[15]$ )

$$
\sigma_{\tau}^{i}, \sigma_{\nu} \in H_{00}^{1 / 2}(\gamma), i=1,2,3
$$

It can be shown that the first relation of (5) holds in the sense

$$
\left\langle\sigma_{\tau}^{i}, \psi\right\rangle_{1 / 2, \gamma}^{00}=0 \quad \forall \psi \in H_{00}^{1 / 2}(\gamma), i=1,2,3,
$$

and the second one holds as

$$
\left\langle\sigma_{\nu}, \psi\right\rangle_{1 / 2, \gamma}^{00} \leq 0 \quad \forall \psi \in H_{00}^{1 / 2}(\gamma), \psi \geq 0
$$

where $\left\langle\cdot, \cdot \operatorname{rangle} e_{1 / 2, \gamma}^{00}\right.$ means a duality pairing between $H_{00}^{-1 / 2}(\gamma)$ and $H_{00}^{1 / 2}(\gamma)$. Condition (6) is fulfilled as follows

$$
\left\langle\sigma_{\nu},\left(u^{+}-\rho_{0}\right) \cdot \nu\right\rangle_{1 / 2, \gamma}^{00}=0,
$$

and the relation (7) holds in the sense

$$
\langle\sigma \nu, \rho\rangle_{1 / 2, \Xi}=-\int_{\omega} F \cdot \rho \quad \forall \rho \in R(\omega),
$$

where $\langle\cdot, \cdot\rangle_{1 / 2, \Xi}$ stands for a duality pairing between $H^{-1 / 2}(\Xi)$ and $H^{1 / 2}(\Xi)$.

\subsection{Dual problem formulation}

We provide here a dual formulation of the problem (14)-(15). This approach allows us to define $\sigma=\left\{\sigma_{i j}\right\}$ in the domain $\Omega \backslash \bar{\omega}$, and moreover, this solution $\sigma_{i j}$ coincide with $\sigma_{i j}=\sigma_{i j}(u)$ obtained from (14)-(15). Below we provide suitable explanations. First, we write Hooke's law (2) in the inverted form

$$
A^{-1} \sigma=\varepsilon(u) \text { in } \Omega \backslash \bar{\omega} .
$$

Note that the tensor $A^{-1}$ has the properties similar to those of $A$. Consider the space

$$
H=\left\{\sigma=\left\{\sigma_{i j}\right\} \mid \sigma_{i j} \in L^{2}(\Omega \backslash \bar{\omega}), i, j=1,2,3\right\}
$$


and a functional $G$ defined on $H$,

$$
G(\sigma)=\frac{1}{2}\left(A^{-1} \sigma, \sigma\right)_{\Omega \backslash \bar{\omega}} .
$$

Introduce next the set of admissible stresses

$$
M=\{\sigma \in H \mid \text { equations (1) and conditions (5), (7) hold }\} .
$$

Fulfillment of (1) in the definition of $M$ takes place in the distributional sense, and (5), (7) hold in the sense (16)-(18).

Notice that $M$ is convex and weakly closed in the space $H$. Indeed, if $\sigma^{n} \in M$ and

$$
\sigma^{n} \rightarrow \sigma \text { weakly in } H, n \rightarrow \infty \text {, }
$$

we have

$$
-\operatorname{div} \sigma^{n}=F \text { in } \Omega \backslash \bar{\omega}
$$

Thus

$$
-\operatorname{div} \sigma=F \text { in } \Omega \backslash \bar{\omega} .
$$

Since

$$
\sigma_{\tau}^{i n}, \sigma_{\nu}^{n} \text { are bounded in } H_{00}^{-1 / 2}(\gamma), i=1,2,3,
$$

we can assume that as $n \rightarrow \infty$

$$
\sigma_{\tau}^{i n}, \sigma_{\nu}^{n} \rightarrow \sigma_{\tau}^{i}, \sigma_{\nu} \text { weakly in } H_{00}^{-1 / 2}(\gamma), i=1,2,3 .
$$

Consequently, the limit function $\sigma$ satisfies (16)-(18) which, by (20), proves that $M$ is weakly closed.

Now consider the minimization problem

$$
\inf _{\sigma \in M} G(\sigma) .
$$

Solution $\sigma^{0}$ of this problem exists and is unique. It satisfies the variational inequality

$$
\begin{aligned}
\sigma^{0} & \in M, \\
\left(A^{-1} \sigma^{0}, \bar{\sigma}-\sigma^{0}\right)_{\Omega \backslash \bar{\omega}} & \geq 0 \quad \forall \bar{\sigma} \in M .
\end{aligned}
$$

Now we denote by $\sigma=\sigma(u)$ the function found from (2) for the solution $u \in K_{\omega}$ of (14)-(15), and prove that $\sigma^{0}=\sigma$. The following relation holds

$$
G\left(\sigma^{0}\right)=G\left(\sigma^{0}-\sigma\right)+G(\sigma)+\left(A^{-1}\left(\sigma^{0}-\sigma\right), \sigma\right)_{\Omega \backslash \bar{\omega}} .
$$

Introduce the notation

$$
p=\left(A^{-1}\left(\sigma^{0}-\sigma\right), \sigma\right)_{\Omega \backslash \bar{\omega}}
$$


and prove that $p \geq 0$. Indeed, by the Green formula we derive

$$
\begin{aligned}
p & =\int_{\Omega \backslash \bar{\omega}}\left(\sigma_{i j}^{0}-\sigma_{i j}\right) \varepsilon_{i j}(u) \\
& =-\int_{\Omega \backslash \bar{\omega}} \operatorname{div}\left(\sigma^{0}-\sigma\right) \cdot u-\int_{\Xi^{+}}\left(\sigma^{0}-\sigma\right) \nu \cdot u .
\end{aligned}
$$

Since $\sigma^{0}, \sigma$ satisfy (1), this relation implies

$$
p=-\int_{\Xi^{+}}\left(\sigma^{0}-\sigma\right) \nu \cdot u
$$

In its own turn, (25) can be rewritten as

$$
p=\int_{\Xi^{+}}\left(\sigma^{0}-\sigma\right) \nu \cdot u-\int_{\gamma^{+}}\left(\sigma^{0}-\sigma\right) \nu \cdot\left(u-\rho_{0}\right),
$$

where $\rho_{0}$ is the restriction of $u$ to $\omega$. Functions $\sigma, \sigma^{0}$ satisfy also (7), thus, by accounting (5), formula (26) takes the form

$$
p=-\int_{\gamma^{+}}\left(\sigma_{\nu}^{0}-\sigma_{\nu}\right)\left(u-\rho_{0}\right) \cdot \nu .
$$

Moreover, $\sigma, u$ satisfy (6), hence

$$
p=-\int_{\gamma^{+}} \sigma_{\nu}^{0}\left(u-\rho_{0}\right) \cdot \nu .
$$

Since $\sigma^{0} \in M$, by (4), from (27) if follows $p \geq 0$. So we have $G\left(\sigma^{0}-\sigma\right) \geq 0$, $p \geq 0$, and the relation (24) implies

$$
G\left(\sigma^{0}\right) \geq G(\sigma)
$$

By the uniqueness of the solution $\sigma^{0}$ of $(21)$, we obtain $\sigma^{0}=\sigma$ what is needed.

\subsection{Passage from elastic inclusion to the rigid one}

In fact, the problem (1)-(7) can be viewed as a limit problem for a family of elastic crack problems formulated in the domain $\Omega_{\gamma}$. This means that we can construct a family of problems with a positive parameter $\lambda$ such that for any fixed $\lambda>0$ the problem describes an equilibrium state for elastic body occupying the domain $\Omega_{\gamma}$ with the crack $\gamma$. When $\lambda \rightarrow 0$ we expect to obtain a rigid inclusion $\omega$ so that any point $x \in \omega$ has a displacement $\rho_{0}(x)$, 
$\rho_{0} \in R(\omega)$. In what follows we prove the above statement. Introduce the tensor $A^{\lambda}=\left\{a_{i j k l}^{\lambda}\right\}, i, j, k, l=1,2,3$,

$$
a_{i j k l}^{\lambda}=\left\{\begin{aligned}
a_{i j k l} & \text { in } \Omega \backslash \bar{\omega} \\
\lambda^{-1} a_{i j k l} & \text { in } \omega,
\end{aligned}\right.
$$

and consider the following problem. In the domain $\Omega_{\gamma}$, we have to find functions $u^{\lambda}=\left(u_{1}^{\lambda}, u_{2}^{\lambda}, u_{3}^{\lambda}\right), \sigma^{\lambda}=\left\{\sigma_{i j}^{\lambda}\right\}, i, j=1,2,3$, such that

$$
\begin{aligned}
-\operatorname{div} \sigma^{\lambda}=F & \text { in } \quad \Omega_{\gamma}, \\
\sigma^{\lambda}-A^{\lambda} \varepsilon\left(u^{\lambda}\right)=0 & \text { in } \quad \Omega_{\gamma}, \\
u^{\lambda}=0 & \text { on } \quad \Gamma, \\
{\left[u^{\lambda}\right] \cdot \nu \geq 0,\left[\sigma_{\nu}^{\lambda}\right]=0, \sigma_{\nu}^{\lambda}[u] \cdot \nu=0 } & \text { on } \gamma, \\
\sigma_{\nu}^{\lambda} \leq 0, \sigma_{\tau}^{\lambda}=0 & \text { on } \gamma^{ \pm} .
\end{aligned}
$$

Here we use notations of the previous section, and $[v]=v^{+}-v^{-}$is a jump of $v$ on $\gamma$, where \pm fit positive and negative crack faces $\gamma^{ \pm}$with respect to $\nu$.

For any fixed $\lambda>0$ the problem (28)-(32) is well known (see [15], [16]). It admits a variational formulation. Indeed, introduce the set of admissible displacements

$$
K=\left\{v \in H_{\Gamma}^{1}\left(\Omega_{\gamma}\right)^{3} \mid[v] \cdot \nu \geq 0 \text { on } \gamma\right\}
$$

where

$$
H_{\Gamma}^{1}\left(\Omega_{\gamma}\right)=\left\{v \in H^{1}\left(\Omega_{\gamma}\right) \mid v=0 \text { on } \Gamma\right\} .
$$

There exists a unique solution $u^{\lambda}$ of the problem

$$
\inf _{v \in K}\left\{\frac{1}{2}\left(\sigma^{\lambda}(v), \varepsilon(v)\right)_{\Omega_{\gamma}}-(F, v)_{\Omega_{\gamma}}\right\}
$$

with $\sigma^{\lambda}(v)$ found via (29) for $u^{\lambda}=v$. Solution $u^{\lambda}$ satisfies the variational inequality

$$
\begin{gathered}
u^{\lambda} \in K, \\
\left(\sigma^{\lambda}\left(u^{\lambda}\right), \varepsilon\left(\bar{u}-u^{\lambda}\right)\right)_{\Omega_{\gamma}} \geq\left(F, \bar{u}-u^{\lambda}\right)_{\Omega_{\gamma}} \forall \bar{u} \in K .
\end{gathered}
$$

By the convexity of the functional in (33) with respect to $u$, the problems (33) and (34)-(35) are equivalent. Moreover, all relations (28)-(32) follow from (34)-(35), and conversely, from (28)-(32) it follows (34)-(35).

Below we justify a passage to the limit as $\lambda \rightarrow 0$ in (34)-(35). Substitute $\bar{u}=0, \bar{u}=2 u^{\lambda}$ as test functions in (35), and sum up the relations obtained. It provides the equality

$$
\left(\sigma^{\lambda}\left(u^{\lambda}\right), \varepsilon\left(u^{\lambda}\right)\right)_{\Omega_{\gamma}}=\left(F, u^{\lambda}\right)_{\Omega_{\gamma}} .
$$


Assuming that $\lambda \in\left(0, \lambda_{0}\right)$, from (36) we obtain

$$
\begin{aligned}
\left\|u^{\lambda}\right\|_{H_{\Gamma}^{1}\left(\Omega_{\gamma}\right)^{3}} & \leq c_{1}, \\
\frac{1}{\lambda} \int_{\omega} a_{i j k l} \varepsilon_{k l}\left(u^{\lambda}\right) \varepsilon_{i j}\left(u^{\lambda}\right) & \leq c_{2}
\end{aligned}
$$

with constants $c_{1}, c_{2}$ being uniform with respect to $\lambda \in\left(0, \lambda_{0}\right)$. Choosing a subsequence, if necessary, it can be assumed as $\lambda \rightarrow 0$

$$
u^{\lambda} \rightarrow u \text { weakly in } H_{\Gamma}^{1}\left(\Omega_{\gamma}\right)^{3} .
$$

Then by (38)

$$
\varepsilon_{i j}(u)=0 \text { in } \omega, i, j=1,2,3 \text {. }
$$

This means that a function $\rho_{0}$ exists such that

$$
u=\rho_{0} \text { in } \omega ; \rho_{0} \in R(\omega) .
$$

Since $u^{\lambda}$ converge weakly in $H_{\Gamma}^{1}\left(\Omega_{\gamma}\right)^{3}$, the limit function $u$ satisfies the inequality

$$
\left(u^{+}-\rho_{0}\right) \cdot \nu \geq 0 \text { on } \gamma \text {. }
$$

In particular, $u \in K_{\omega}$.

Let us take any fixed element $\bar{u} \in K_{\omega}$. Then, there exists $\rho \in R(\omega)$ such that $\bar{u}=\rho$ in $\omega$, and $\bar{u}$ can be taken as a test function in (35). In such a case, inequality (35) implies

$$
\left(\sigma^{\lambda}\left(u^{\lambda}\right), \varepsilon\left(\bar{u}-u^{\lambda}\right)\right)_{\Omega_{\gamma}} \geq\left(F, \bar{u}-u^{\lambda}\right)_{\Omega_{\gamma}} .
$$

By accounting $A^{\lambda}=A$ in $\Omega \backslash \bar{\omega}$, we can pass to the limit in (39) as $\lambda \rightarrow 0$ which implies

$$
\begin{aligned}
u & \in K_{\omega}, \\
(\sigma(u), \varepsilon(\bar{u}-u))_{\Omega_{\gamma} \backslash \bar{\omega}} & \geq(F, \bar{u}-u)_{\Omega_{\gamma}} \forall \bar{u} \in K_{\omega},
\end{aligned}
$$

what is precisely (14)-(15). Hence a passage from the elastic inclusion to the rigid one is justified. We formulate the proved result as follows

Theorem 2.1 The solution $u^{\lambda}$ of the problem (34)-(35) weakly converge in $H_{\Gamma}^{1}\left(\Omega_{\gamma}\right)^{3}$ to the solution $u$ of the problem (14)-(15).

Observe that there is no limit for the stress tensor $\sigma^{\lambda}$ as $\lambda \rightarrow 0$ in $\omega$. It is interesting to compare the above passage to the limit with the fictitious domain approach in contact problems, see [14]. 


\section{Shape differentiability of the energy functional}

First of all, we recall the result on the Hadamard differentiability of the metric projection on polyhedric convex sets in Hilbert spaces due to F. Mignot and A. Haraux , adapted to the weak formulation of our problem [29], [7], given by variational inequality (14)-(15).

Theorem 3.1 Let there be given the right-hand side $F_{t}=F+$ th of variational inequality (14)-(15), then the unique solution $u_{t} \in K_{\omega}$ is Lipschitz continuous

$$
\left\|u_{t}-u\right\|_{H^{1}\left(\Omega_{\gamma}\right)} \leq C t
$$

and conically differentiable in $H^{1}\left(\Omega_{\gamma}\right)$, that is, for $t>0, t$ small enough,

$$
u_{t}=u+t Q+o(t)
$$

where the conical differential solves the variational inequality

$$
\begin{aligned}
Q & \in S_{K}(u), \\
(\sigma(O), \varepsilon(\bar{u}-Q))_{\Omega_{\gamma} \backslash \bar{\omega}} & \geq(h, \bar{u}-Q)_{\Omega_{\gamma}} \quad \forall \bar{u} \in S_{K}(u) .
\end{aligned}
$$

The remainder converges to zero

$$
\frac{1}{t}\|o(t)\|_{H^{1}\left(\Omega_{\gamma}\right)} \rightarrow 0
$$

uniformly with respect to the direction $h$ on the compact sets of the dual space $\left(H_{\Gamma}^{1, \omega}\left(\Omega_{\gamma}\right)\right)^{*}$, i.e., $Q$ is the Hadamard directional derivative of the solution to the variational inequality with respect to the right-hand side.

To complete the above statement, we need the description of the convex cone $S_{K}(u)$,

$$
S_{K}(u)=\left\{v \in H_{\Gamma}^{1, \omega}\left(\Omega_{\gamma}\right) \mid\left(v^{+}-v^{-}\right) \cdot \nu \geq 0 \text { on } \gamma_{0} ;(\sigma(u), \varepsilon(v))_{\Omega_{\gamma} \backslash \bar{\omega}}=(F, v)_{\Omega_{\gamma}}\right\}
$$

where $\gamma_{0}=\left\{x \in \gamma \mid\left(u^{+}-\rho_{0}\right) \cdot \nu=0\right\}$.

We show that the energy functional is shape differentiable with respect to the crack length, which seems to be a new result in the fracture mechanics for the specific problem. To this end we need the notation.

Shape sensitivity analysis in the hold-all-domain. We denote $\Omega_{0}:=\Omega_{\gamma}$, and $\mathcal{D}=D \cup \omega$, so $D$ is our hold-all-domain for velocity vector fields, in addition we have the inclusion $\bar{\Omega} \subset \mathcal{D}$. We assume that the boundary $\partial D=\partial \mathcal{D} \cup \partial \omega$ is smooth, and that admissible vector fields for the velocity method of shape optimization satisfies the Nagumo condition

$$
V \cdot n=0 \quad \text { on } \quad \partial D \text {. }
$$


The above condition guarantee the following properties of the mapping $T_{t}(V)$, the support of any admissible vector field $V$ is disjoint with $\omega, V$ is tangent on the boundary $\partial \omega$, we can also deform $\gamma \subset \partial \Omega$ in the tangential direction, therefore, to move the crack boundary on the surface $\partial \omega$. Hence, we denote $\gamma_{t}=T_{t}(V)(\gamma)$ and $\Gamma_{t}=T_{t}(V)(\Gamma)$ and we observe that the boundary of rigid inclusion is invariant for admissible transformations $T_{t}(V)$, it means that $\omega=$ $T_{t}(\omega)$.

Variable domain setting. In order to perform the shape sensitivity analysis of the energy functional $J\left(\Omega_{\gamma}\right)$ we transport the problem defined in the variable domain $\Omega_{t}=T_{t}\left(\Omega_{\gamma}\right)$ to the fixed domain $\Omega_{\gamma}=\Omega_{\gamma}$. To this end we need also a change of the unknown solution to the variational inequality, in order to make the convex cone independent of the parameter $t$. First, we define the problem in variable domain $\Omega_{t}=T_{t}(V)\left(\Omega_{\gamma}\right)$, so we look for the minimizer $u_{t} \in K_{t}$ defined by the variational inequality

$$
\left(\sigma\left(u_{t}\right), \varepsilon\left(v-u_{t}\right)\right)_{\Omega_{t} \backslash \bar{\omega}}-\left(F, v-u_{t}\right)_{\Omega_{t}}+\geq 0 \forall v \in K_{t},
$$

where

$$
K_{t}=\left\{v \in H_{\Gamma_{t}}^{1, \omega}\left(\Omega_{t}\right) \mid\left(v^{+}-v^{-}\right) \cdot \nu_{t} \geq 0 \text { on } \gamma_{t}\right\}
$$

Now, we perform the shape sensitivity analysis in exactly the same way as it is described in [29] for the Signorini problems. We use also the same notation which is introduced in [29] for the sensitivity analysis of variational inequalities with the polyhedric convex cones, this is the case of the convex set (44). However, the results presented here seem to be new, since the model of the crack with nonpenetration condition is new in this setting. We derive the form of the directional derivative of the energy functional with respect to the crack length, which a generalization of the results given in [17] for the crack located inside of an elastic body.

In order to assure the fixed domain setting for the transported problem we introduce the new unknown solution to the modified variational inequality $z^{t}=D T_{t}^{-1} \cdot u_{t} \circ T_{t}$, and we obtain that $z^{t} \in K$ solves the variational inequality

$$
\left(\sigma^{t}\left(z^{t}\right), \varepsilon^{t}\left(v-z^{t}\right)\right)_{\Omega_{\gamma} \backslash \bar{\omega}}-\left(F^{t}, v-z^{t}\right)_{\Omega_{\gamma}} \geq 0 \forall v \in K
$$

with the shape functional of the form

$$
J\left(\Omega_{t}\right)=\frac{1}{2}\left(\sigma^{t}\left(z^{t}\right), \varepsilon^{t}\left(z^{t}\right)\right)_{\Omega_{\gamma} \backslash \bar{\omega}}-\left(F^{t}, z^{t}\right)_{\Omega_{\gamma}} .
$$


The expressions for $F^{t}, \sigma^{t}(v)$ and $\varepsilon^{t}(v)$ are given by

$$
\begin{gathered}
F^{t}=\operatorname{det}\left(D T_{t}\right)^{*} D T_{t} \cdot\left(F \circ T_{t}\right) \\
\sigma^{t}(v)=\operatorname{det}\left(D T_{t}\right) A \varepsilon^{t}(v) \\
\varepsilon^{t}(v)=\frac{1}{2}\left\{D\left(D T_{t} \cdot v\right) \cdot D T_{t}^{-1}+{ }^{*} D T_{t}^{-1} \cdot{ }^{*}\left(D\left(D T_{t} \cdot v\right)\right)\right\}
\end{gathered}
$$

where $D T_{t}$ is the Jacobian matrix of the transformation $T_{t},{ }^{*} D T_{t}$ denotes the transposed matrix, $D T_{t}^{-1}$ its inverse, and $\operatorname{det}\left(D T_{t}\right)$ its determinant.

Since the convex set $K_{t}$ is a cone, it follows from the variational inequality that we have the following equality

$$
\left(\sigma^{t}\left(z^{t}\right), \varepsilon^{t}\left(z^{t}\right)\right)_{\Omega_{\gamma} \backslash \bar{\omega}}=\left(F^{t}, z^{t}\right)_{\Omega_{\gamma}}
$$

therefore, the equivalent form for the energy shape functional looks like that

$$
J\left(\Omega_{t}\right)=-\frac{1}{2}\left(\sigma^{t}\left(z^{t}\right), \varepsilon^{t}\left(z^{t}\right)\right)_{\Omega_{\gamma} \backslash \bar{\omega}}=-\frac{1}{2}\left(F^{t}, z^{t}\right)_{\Omega_{\gamma}} .
$$

The structure of formulae in (51) is useful for the derivation with respect to the shape parameter $t$ at $t=0$.

Theorem 3.2 The solutions to variational inequality (45) are shape differentiable in the sense of Hadamard, and the mateial derivatives $\dot{z} \in \mathcal{S}_{K}(u)$ are given by the following variational inequality

$(\sigma(\dot{z}), \varepsilon(v-\dot{z}))_{\Omega_{\gamma} \backslash \bar{\omega}}+(\dot{\sigma}(u), \varepsilon(v-\dot{z}))_{\Omega_{\gamma} \backslash \bar{\omega}}+(\dot{\varepsilon}(u), \sigma(v-\dot{z}))_{\Omega_{\gamma} \backslash \bar{\omega}} \geq(\dot{F}, v-\dot{z})_{\Omega_{\gamma}}$

for all test functions in the convex cone $v \in \mathcal{S}_{K}(u)$.

Corollary 3.3 The shape derivative of the energy functional is given by the expression,

$$
d J\left(\Omega_{\gamma} ; V\right)=-\frac{1}{2}\left\{(\dot{\sigma}(u), \varepsilon(u))_{\Omega_{\gamma} \backslash \bar{\omega}}+(\sigma(u), \dot{\varepsilon}(u))_{\Omega_{\gamma} \backslash \bar{\omega}}+2(\sigma(\dot{z}), \varepsilon(u))_{\Omega_{\gamma} \backslash \bar{\omega}}\right\}
$$

where $u \in K$ solves variational inequality (45), and $\dot{z} \in \mathcal{S}_{K}(u)$ solves the variational inequality for material derivatives (52).

The expressions for $\dot{\sigma}(v)$ and $\dot{\varepsilon}(v)$ are given by

$$
\begin{gathered}
\dot{\sigma}(v)=\operatorname{div} V A \varepsilon(v)+A \dot{\varepsilon}(v) \\
\dot{\varepsilon}(v)=\frac{1}{2}\left\{D(D V \cdot v)+{ }^{*}(D(D V \cdot v))-D v \cdot D V-{ }^{*} D V \cdot{ }^{*} D v\right\} .
\end{gathered}
$$


Therefore, we have the shape derivative of the energy functional with respect to the crack length, this formula is new and could be used in crack propagation analysis.

\section{Topological asymptotic analysis}

The topological derivative introduced in [26] quantifies the sensitivity of a given shape functional with respect to the introduction of a non-smooth perturbation (hole, inclusion, source term, for instance) in a ball $B_{\delta}\left(x_{0}\right) \subset \Omega$ of radius $\delta>0$ and center at $x_{0} \in \Omega$, that is $B_{\delta}\left(x_{0}\right)=\left\{x \in \mathbb{R}^{3}:\left\|x-x_{0}\right\|<\delta\right\}$, $\overline{B_{\delta}\left(x_{0}\right)}$ is the closure of $B_{\delta}\left(x_{0}\right)$. Therefore, this derivative can be seen as a first order correction on the shape functional $\mathcal{J}(\Omega)$ to estimate $\mathcal{J}\left(\Omega_{\delta}\right)$, where $\Omega_{\delta}$ is the perturbed domain. Thus, we have the following topological asymptotic expansion for functional $\mathcal{J}[?]$,

$$
\mathcal{J}\left(\Omega_{\delta}\right)=\mathcal{J}(\Omega)+f(\delta) D_{T}\left(x_{0}\right)+o(f(\delta))
$$

where $f(\delta)$ is a positive function that decreases monotonically such that $f(\delta) \rightarrow 0$ when $\delta \rightarrow 0^{+}$and the term $D_{T}\left(x_{0}\right)$ is defined as the topological derivative of $\mathcal{J}$. Then, from (56) we have that the classical definition of the topological derivative is given by $[24,26]$

$$
D_{T}\left(x_{0}\right)=\lim _{\delta \rightarrow 0} \frac{\mathcal{J}\left(\Omega_{\delta}\right)-\mathcal{J}(\Omega)}{f(\delta)}=\lim _{\delta \rightarrow 0} \frac{1}{f^{\prime}(\delta)} \frac{d}{d \delta} \mathcal{J}\left(\Omega_{\delta}\right)
$$

Therefore, the topological derivative has been successfully applied in the context of topology optimization $[1,4]$, inverse problems $[2,6]$ and image processing $[3,13,20]$. Concerning the theoretical development of the topological asymptotic analysis, the reader may refer to [23], for instance.

In our particular case, we consider a perturbation on the domain given by the nucleation of a small elastic inclusion with Young modulus $E_{\eta}=\eta E$, where $E$ is the Young modulus of the bulk material and $\eta \in[0, \infty)$ represents the contrast. We assume that there is a small elastic inclusion $B_{\delta}\left(x_{0}\right)$ in the elastic region $\Omega_{\omega}=\Omega \backslash \bar{\omega}$. If the elastic inclusion becomes a cavity, it is denoted by $\omega_{\delta}=B_{\delta}\left(x_{0}\right)$. The cavity can be obtained from the elastic inclusion by the limit passage $\eta \rightarrow 0$. In the case of elastic inclusion the elastic region $\Omega_{\omega}$ is decomposed into two disjoint parts $\Omega_{\omega} \backslash \overline{B_{\delta}\left(x_{0}\right)}$ and $B_{\delta}\left(x_{0}\right)$ with different material properties, namely $E$ and $\eta E$, respectively. The other limit passage with the contrast $\eta \rightarrow \infty$ results in the small rigid inclusion $\omega_{\delta}=B_{\delta}\left(x_{0}\right)$. See Fig. (2). We are also interested in the topological asymptotic expansion of the 

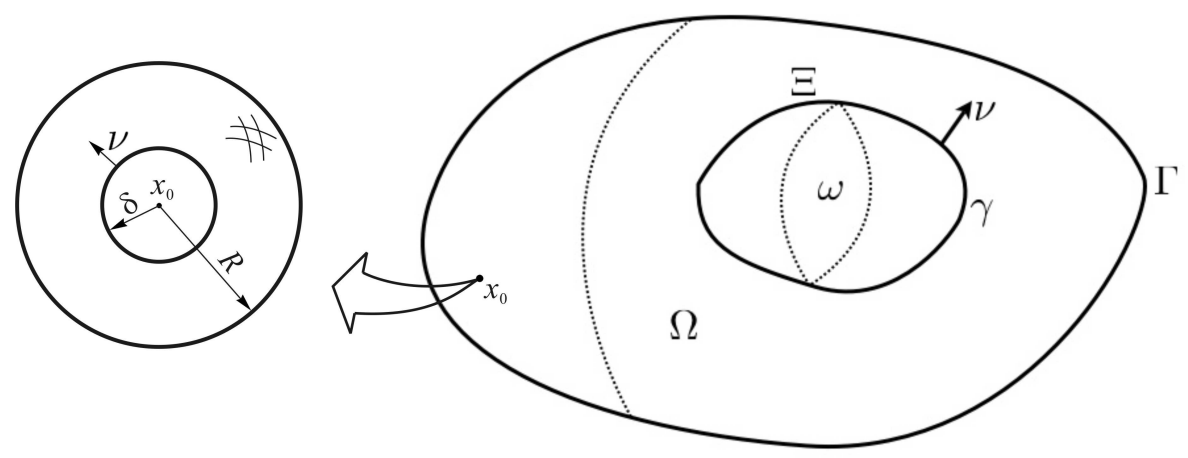

Fig. 2. Domain $\Omega$ with rigid inclusion $\omega$ and an elastic inclusion $B_{\delta}\left(x_{0}\right)$.

energy shape functional of the form

$$
\Pi_{\delta}(v)=\frac{1}{2}(\sigma(v), \varepsilon(v))_{\Omega_{\omega} \backslash \overline{B_{\delta}\left(x_{0}\right)}}+\frac{1}{2}(\sigma(v), \varepsilon(v))_{B_{\delta}\left(x_{0}\right)}-(F, v)_{\Omega_{\gamma}},
$$

where we have to find function $v=u_{\delta}$ such that

$$
\begin{aligned}
-\operatorname{div} \sigma=F & \text { in } \quad \Omega \backslash \bar{\omega}, \\
\sigma-A \varepsilon\left(u_{\delta}\right)=0 & \text { in } \quad \Omega_{\omega} \backslash \overline{B_{\delta}\left(x_{0}\right)}, \\
\sigma-A_{\eta} \varepsilon\left(u_{\delta}\right)=0 & \text { in } \quad B_{\delta}\left(x_{0}\right), \\
{\left[u_{\delta}\right]=0 } & \text { on } \quad \partial B_{\delta}\left(x_{0}\right) \\
{[\sigma] \nu=0 } & \text { on } \partial B_{\delta}\left(x_{0}\right) \\
u_{\delta}=0 & \text { on } \Gamma, \\
\left(u_{\delta}-\rho_{0}\right) \cdot \nu \geq 0 & \text { on } \gamma^{+}, \\
\sigma_{\tau}=0, \sigma_{\nu} \leq 0 & \text { on } \gamma^{+}, \\
\sigma_{\nu}\left(u_{\delta}-\rho_{0}\right) \cdot \nu=0 & \text { on } \gamma^{+}, \\
-\int_{\Xi} \sigma \nu \cdot \rho=\int_{\omega} F \cdot \rho & \forall \rho \in R(\omega) .
\end{aligned}
$$

with $A$ such as before and $A_{\eta}=\eta A$ (since $E_{\eta}$ is the Young modulus of the inclusion).

\subsection{Domain decomposition}

Since the problem is non-linear, let us introduce a domain decomposition given by $\Omega_{R}=\Omega_{\omega} \backslash \overline{B_{R}\left(x_{0}\right)}$, where $B_{R}\left(x_{0}\right)$ is a ball of radius $R>\delta$ and center at $x_{0} \in \Omega$, that is $B_{R}\left(x_{0}\right)=\left\{x \in \mathbb{R}^{3}:\left\|x-x_{0}\right\|<R\right\}, \overline{B_{R}\left(x_{0}\right)}$ is the closure of $B_{R}\left(x_{0}\right)$, as shown in Fig. (2). For the sake of simplicity, we assume that $F=0$ in $B_{R}\left(x_{0}\right)$. Thus, we have the following linear elasticity system defined 
in $B_{R}\left(x_{0}\right)$ with an inclusion $B_{\delta}\left(x_{0}\right)$ inside

$$
\begin{array}{rll}
-\operatorname{div} \sigma=0 & \text { in } & B_{R}\left(x_{0}\right), \\
\sigma-A \varepsilon\left(w_{\delta}\right)=0 & \text { in } & B_{R}\left(x_{0}\right) \backslash \overline{B_{\delta}\left(x_{0}\right)}, \\
\sigma-A_{\eta} \varepsilon\left(w_{\delta}\right)=0 & \text { in } & B_{\delta}\left(x_{0}\right), \\
w_{\delta}=v & \text { on } & \partial B_{R}\left(x_{0}\right), \\
{\left[w_{\delta}\right]=0} & \text { on } & \partial B_{\delta}\left(x_{0}\right), \\
{[\sigma] \nu=0} & \text { on } & \partial B_{\delta}\left(x_{0}\right) .
\end{array}
$$

We are interested in the Steklov-Poincaré operator on $\partial B_{R}$, that is

$$
\mathcal{A}_{\delta}: v \in H^{1 / 2}\left(\partial B_{R}\right) \mapsto \sigma\left(w_{\delta}\right) \nu \in H^{-1 / 2}\left(\partial B_{R}\right) .
$$

Then we have $\sigma\left(u_{R}\right) \nu=\mathcal{A}_{\delta}\left(u_{R}\right)$ on $\partial B_{R}$, where $u_{R}$ is solution of the variational inequality in $\Omega_{R}$, that is

$$
u_{R} \in K_{\omega}: a_{\Omega_{R}}\left(u_{R}, \varphi-u_{R}\right) \geq\left(F, \varphi-u_{R}\right)_{\Omega_{\gamma} \backslash \overline{B_{R}\left(x_{0}\right)}} \quad \forall \varphi \in K_{\omega}
$$

and the bilinear form $a_{\Omega_{R}}$ is such that

$$
a_{\Omega_{R}}(u, \varphi)=\int_{\Omega_{R}} \sigma(u) \cdot \varepsilon(\varphi)+\int_{\partial B_{R}} \mathcal{A}_{\delta}(u) \cdot \varphi .
$$

Finally, in the disk $B_{R}\left(x_{0}\right)$ we have

$$
\int_{B_{R} \backslash \overline{B_{\delta}}} \sigma(w) \cdot \varepsilon(w)+\int_{B_{\delta}} \sigma(w) \cdot \varepsilon(w)=\int_{\partial B_{R}} \mathcal{A}_{\delta}(w) \cdot w
$$

where $w=w_{\delta}$ is the solution of the elasticity system in the disk (69)-(74) or equivalently solution of the following variational problem

$$
w_{\delta} \in W: \int_{B_{R} \backslash \overline{B_{\delta}}} \sigma\left(w_{\delta}\right) \cdot \varepsilon(\varphi)+\int_{B_{\delta}} \sigma\left(w_{\delta}\right) \cdot \varepsilon(\varphi)=0 \quad \forall \varphi \in W_{0},
$$

with $W$ and $W_{0}$ such that

$$
\begin{aligned}
W & =\left\{w \in H^{1}\left(B_{R}\right)^{3} \mid[w]=0 \text { on } \partial B_{\delta}, w=v \text { on } \partial B_{R}\right\} \\
W_{0} & =\left\{\varphi \in H^{1}\left(B_{R}\right)^{3} \mid[\varphi]=0 \text { on } \partial B_{\delta}, \varphi=0 \text { on } \partial B_{R}\right\} .
\end{aligned}
$$

\subsection{Shape sensitivity analysis of the energy functional}

Let us introduced the energy-based shape functional defined in the disk $B_{R}\left(x_{0}\right)$, that is

$$
\mathcal{E}_{\delta}\left(w_{\delta}\right):=\frac{1}{2} \int_{B_{R} \backslash \overline{B_{\delta}}} \sigma\left(w_{\delta}\right) \cdot \varepsilon\left(w_{\delta}\right)+\frac{1}{2} \int_{B_{\delta}} \sigma\left(w_{\delta}\right) \cdot \varepsilon\left(w_{\delta}\right) .
$$


We need to calculate

$$
\begin{aligned}
\frac{d}{d \delta} \mathcal{E}_{\delta}\left(w_{\delta}\right) & =\int_{B_{R} \backslash \overline{B_{\delta}}} \sigma\left(w_{\delta}\right) \cdot \varepsilon\left(\dot{w}_{\delta}\right)+\int_{B_{\delta}} \sigma\left(w_{\delta}\right) \cdot \varepsilon\left(\dot{w}_{\delta}\right) \\
& +\int_{B_{R} \backslash \overline{B_{\delta}}} \Sigma\left(w_{\delta}\right) \cdot \nabla V+\int_{B_{\delta}} \Sigma\left(w_{\delta}\right) \cdot \nabla V
\end{aligned}
$$

which was obtained using the Reynold's transport theorem and the concept of material derivatives of spacial fields [10,29]. Some of the terms in (84) require explanation. Vector $V$ represents the shape change velocity field defined on the disk $B_{R}\left(x_{0}\right)$ and such that $V=0$ on $\partial B_{R}$ and $V=\nu$ on $\partial B_{\delta}$. Thus, $\dot{w}_{\delta} \in W_{0}$ is the material (total) derivative with respect to $\delta$. Finally, the Eshelby energy-momentum tensor $\Sigma$ takes the form $[5,11]$

$$
\Sigma\left(w_{\delta}\right):=\frac{1}{2} \sigma\left(w_{\delta}\right) \cdot \varepsilon\left(w_{\delta}\right) \mathrm{I}-\left(\nabla w_{\delta}\right)^{T} \sigma\left(w_{\delta}\right) .
$$

Since $\dot{w}_{\delta} \in W_{0}$ and considering that $\Sigma\left(w_{\delta}\right)$ is a free-divergence tensor field $\left(\operatorname{div} \Sigma\left(w_{\delta}\right)=0\right)$, the shape derivative of the energy functional becomes

$$
\frac{d}{d \delta} \mathcal{E}_{\delta}\left(w_{\delta}\right)=-\int_{\partial B_{\delta}}\left[\Sigma\left(w_{\delta}\right)\right] \nu \cdot \nu
$$

\subsection{Topological derivatives calculation}

By introducing (85) in (57), we have

$$
D_{T}\left(x_{0}\right)=-\lim _{\delta \rightarrow 0} \frac{1}{f^{\prime}(\delta)} \int_{\partial B_{\delta}}\left[\Sigma\left(w_{\delta}\right)\right] \nu \cdot \nu .
$$

4.3.1 Topological derivative of the energy functional in three spatial dimensions for a small cavity

In the three spatial dimensions we consider the particular case associated to the energy change due to the nucleation of a spherical cavity. Thus, for the convenience of the reader we recall here the results derived in $[8,12,25]$ for the three dimensional elasticity case.

Theorem 4.1 Let us consider the contrast $\eta \rightarrow 0$. Thus, the elastic inclusion degenerates to a spherical cavity with homogeneous Neumann boundary condition. In this case, the energy shape functional admits for $\delta \rightarrow 0$ the following topological asymptotic expansion

$$
\Pi_{\delta}\left(u_{\delta}\right)=\Pi(u)+\pi \delta^{3} D_{T}\left(x_{0}\right)+o\left(\delta^{3}\right),
$$


with the topological derivative $D_{T}\left(x_{0}\right)$ given by

$$
D_{T}\left(x_{0}\right)=\mathbb{H} \sigma\left(u\left(x_{0}\right)\right) \cdot \varepsilon\left(u\left(x_{0}\right)\right) \quad \forall x_{0} \in \Omega \backslash \bar{\omega},
$$

where $u$ is solution of the variational inequality (14)-(15) and $\mathbb{H}$ is a forthorder tensor defined as

$$
\mathbb{H}=\frac{1-v}{7-5 v}\left(10 \mathbb{I}-\frac{1-5 v}{1-2 v} \mathrm{I} \otimes \mathrm{I}\right)
$$

4.3.2 Topological derivative of the energy functional in two spatial dimensions for a small inclusion

In two spatial directions we derive also an exterior expansion for the solutions of the variational inequality. Therefore, the result obtained is more precise compared to the general case of the cavity in three spatial dimensions. First, we repeat the model description, and then we develop the asymptotic analysis in linear elasticity to derive the equivalent form of perturbation of the bilinear form.

Since in this Section we are dealing with a two dimensional elasticity problem, then the domain $\Omega \subset \mathbb{R}^{2}$. Thus, all indices introduced in the Section 2 take values from 1 to 2 , instead of 1 to 3 . In the particular case of plane stress, the Lamé coefficient $l=l^{*}$, where

$$
l^{*}=\frac{v E}{1-v^{2}} .
$$

In addition, the crack $\gamma$ is represented now by a smooth 1D curve described as

$$
x_{i}=x_{i}(y), \quad y \in D, i=1,2,
$$

with bounded domain $D \subset \mathbb{R}$. The space $R(\omega)$ of infinitesimal rigid displacements is redefined simply by setting

$$
B=\left(\begin{array}{cc}
0 & b \\
-b & 0
\end{array}\right) \quad \text { and } \quad C=\left(c^{1}, c^{2}\right) ; \quad b, c^{i}=\text { const }, i=1,2 \text {. }
$$

The displacement field $u=\left(u_{1}, u_{2}\right) ; u=\rho_{0}$ in $\omega ; \rho_{0} \in R(\omega)$; and in the domain $\Omega \backslash \bar{\omega}$ we have to find the stress tensor components $\sigma=\left\{\sigma_{i j}\right\}$, solution of (1)-(7) in $\Omega \subset \mathbb{R}^{2}$ for $i, j=1,2$. Hence, all definitions and results presented in the previous Sections hold.

We use the existence of the asymptotic expansions for $w_{\delta}$, solution of the elasticity system (69)-(74) now defined in the disk $B_{R}\left(x_{0}\right) \subset \mathbb{R}^{2}$, in the neigh- 


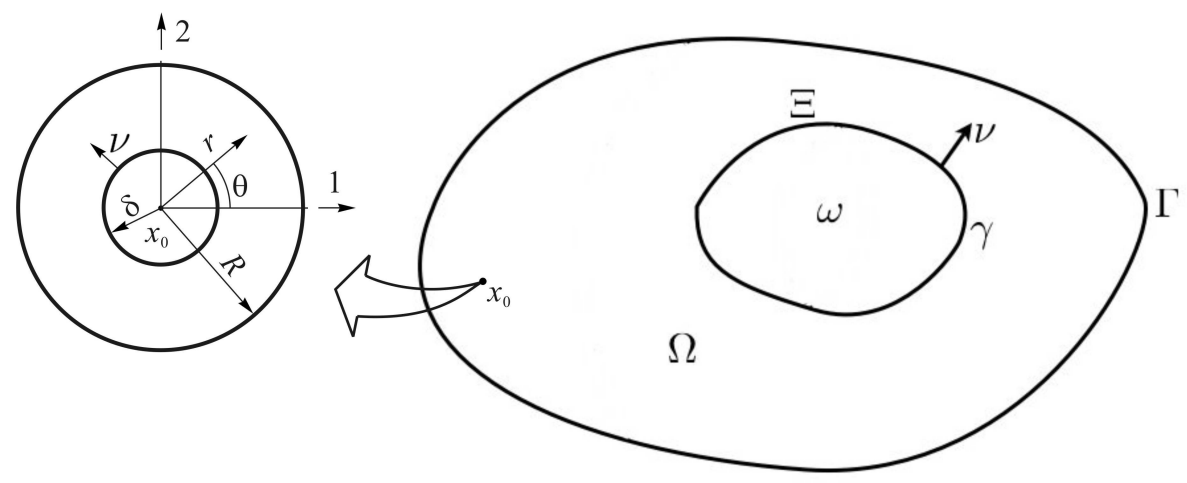

Fig. 3. Domain $\Omega$ with rigid inclusion $\omega$ and an elastic inclusion $B_{\delta}\left(x_{0}\right)$.

borhood of $B_{\delta}\left(x_{0}\right)$, namely

$$
w_{\delta}(x)=w_{0}(x)+w^{\infty}(x)+o(\delta) .
$$

In addition, $w^{\infty}$ is proportional to $\delta,\left\|w^{\infty}\right\|_{\mathbb{R}^{2}}=O(\delta)$, on the surface $\partial B_{\rho}$ of the ball. The expansion of $\sigma\left(w_{\delta}\right)$ corresponding to (69)-(74) has the form

$$
\sigma\left(w_{\delta}(x)\right)=\sigma^{\infty}\left(w_{0}\left(x_{0}\right), x\right)+O(\delta) .
$$

where $\sigma^{\infty}$ is the stress distribution around a circular inclusion in an infinity medium and $w_{0}$ is solution of the elasticity system (69)-(74) defined in the disk $B_{R}\left(x_{0}\right) \subset \mathbb{R}^{2}$ for $\delta=0$. Thus, $\sigma^{\infty}$ can be calculated explicitly, which is given in a polar coordinate system $(r, \theta)$ by:

- for $r \geq \delta$

$$
\begin{aligned}
& \sigma_{r r}^{\infty}(r, \theta)=a\left(1-\frac{1-\eta}{1+\eta \alpha} \frac{\delta^{2}}{r^{2}}\right)+b\left(1-4 \frac{1-\eta}{1+\eta \beta} \frac{\delta^{2}}{r^{2}}+3 \frac{1-\eta}{1+\eta \beta} \frac{\delta^{4}}{r^{4}}\right) \cos 2 \theta \\
& \sigma_{\theta \theta}^{\infty}(r, \theta)=a\left(1+\frac{1-\eta}{1+\eta \alpha} \frac{\delta^{2}}{r^{2}}\right)-b\left(1+3 \frac{1-\eta}{1+\eta \beta} \frac{\delta^{4}}{r^{4}}\right) \cos 2 \theta \\
& \sigma_{r \theta}^{\infty}(r, \theta)=-b\left(1+2 \frac{1-\eta}{1+\eta \beta} \frac{\delta^{2}}{r^{2}}-3 \frac{1-\eta}{1+\eta \beta} \frac{\delta^{4}}{r^{4}}\right) \sin 2 \theta
\end{aligned}
$$

- for $0<r<\delta$

$$
\begin{aligned}
& \sigma_{r r}^{\infty}(r, \theta)=2 \frac{\eta \alpha}{1+\eta \alpha} \frac{a}{1-v}+4 \frac{\eta \beta}{1+\eta \beta} \frac{b}{3-v} \cos 2 \theta \\
& \sigma_{\theta \theta}^{\infty}(r, \theta)=2 \frac{\eta \alpha}{1+\eta \alpha} \frac{a}{1-v}-4 \frac{\eta \beta}{1+\eta \beta} \frac{b}{3-v} \cos 2 \theta \\
& \sigma_{r \theta}^{\infty}(r, \theta)=-4 \frac{\eta \beta}{1+\eta \beta} \frac{b}{3-v} \sin 2 \theta
\end{aligned}
$$

In the above formulas, coefficients $a$ and $b$ are given respectively by

$$
a=\frac{1}{2}\left(\sigma_{1}+\sigma_{2}\right) \quad \text { and } \quad b=\frac{1}{2}\left(\sigma_{1}-\sigma_{2}\right)
$$

where $\sigma_{1,2}$ are the eigenvalues of tensor $\sigma\left(w_{0}\left(x_{0}\right)\right)$. In addition, constants $\alpha$ 
and $\beta$ are respectively given by

$$
\alpha=\frac{1+v}{1-v} \text { and } \beta=\frac{3-v}{1+v} .
$$

The jump condition of the stress field $\sigma\left(w_{\delta}\right)$ can be written as

$$
\left[\sigma\left(w_{\delta}\right)\right] \nu=0 \Rightarrow\left[\sigma_{r r}\left(w_{\delta}\right)\right]=0 \quad \text { and } \quad\left[\sigma_{r \theta}\left(w_{\delta}\right)\right]=0 \text { on } \partial B_{\delta} .
$$

In the same way, the continuity condition of the displacement field $w_{\delta}$ implies

$$
\left[w_{\delta}\right]=0 \Rightarrow\left[\varepsilon_{\theta \theta}\left(w_{\delta}\right)\right]=0 \text { on } \partial B_{\delta}
$$

The Eshelby tensor flux through the boundary of the inclusion is given by

$$
\begin{aligned}
\Sigma\left(w_{\delta}\right) \nu \cdot \nu & =\frac{1}{2} \sigma\left(w_{\delta}\right) \cdot \varepsilon\left(w_{\delta}\right)-\sigma\left(w_{\delta}\right) \nu \cdot\left(\nabla w_{\delta}\right) \nu \\
& =\frac{1}{2}\left(\sigma_{\theta \theta}\left(w_{\delta}\right) \varepsilon_{\theta \theta}\left(w_{\delta}\right)-\sigma_{r r}\left(w_{\delta}\right) \varepsilon_{r r}\left(w_{\delta}\right)\right. \\
& \left.+\sigma_{r \theta}\left(w_{\delta}\right)\left(\partial_{\theta} w_{\delta}^{r}-\partial_{r} w_{\delta}^{\theta}\right)\right) .
\end{aligned}
$$

From the jump and continuity conditions on the boundary $\partial B_{\delta}$ given by $(100$, 101) and considering the constitutive relation (60)-(61) for $l=l^{*}$, the jump of the Eshelby tensor flux in the normal direction results in (see, for instance, $[9])$

$$
\begin{aligned}
{\left[\Sigma\left(w_{\delta}\right)\right] \nu \cdot \nu } & =\frac{1}{2}\left(\left[\sigma_{\theta \theta}\left(w_{\delta}\right)\right] \varepsilon_{\theta \theta}\left(w_{\delta}\right)-\sigma_{r r}\left(w_{\delta}\right)\left[\varepsilon_{r r}\left(w_{\delta}\right)\right]\right. \\
& \left.+2(1-\delta) \sigma_{r \theta}\left(w_{\delta}\right) \varepsilon_{r \theta}\left(w_{\delta}\right)\right)
\end{aligned}
$$

Finally, considering (104) in (86) and also formulas (92)-(96) we can calculate the integral on $\partial B_{\delta}$ explicitly, which allows to identify function $f(\delta)=\pi \delta^{2}$. Then, after calculate the limit $\delta \rightarrow 0$, we obtain the following result:

Theorem 4.2 The energy shape functional admits for $\delta \rightarrow 0$ the following topological asymptotic expansion

$$
\Pi_{\delta}\left(u_{\delta}\right)=\Pi(u)+\pi \delta^{2} D_{T}\left(x_{0}\right)+o\left(\delta^{2}\right),
$$

with the topological derivative $D_{T}\left(x_{0}\right)$ given by

$$
D_{T}\left(x_{0}\right)=\mathbb{H}_{\eta} \sigma\left(u\left(x_{0}\right)\right) \cdot \varepsilon\left(u\left(x_{0}\right)\right) \quad \forall x_{0} \in \Omega \backslash \bar{\omega},
$$


where $u$ is solution of the variational inequality (14)-(15) in $\Omega \subset \mathbb{R}^{2}$ and $\mathbb{H}_{\eta}$ is a forth-order tensor defined as

$$
\mathbb{H}_{\eta}=\frac{1}{4} \frac{(1-\eta)^{2}}{1+\beta \eta}\left(2 \frac{1+\beta}{1-\eta} \mathbb{I}+\frac{\alpha-\beta}{1+\alpha \eta} \mathrm{I} \otimes \mathrm{I}\right)
$$

Corollary 4.3 Let us consider the contrast $\eta \rightarrow 0$. Thus, the elastic inclusion degenerates to a circular cavity with homogeneous Neumann boundary condition and the tensor $\mathbb{H}_{0}$ becomes

$$
\mathbb{H}_{0}=\frac{1}{4}(2(1+\beta) \mathbb{I}+(\alpha-\beta) \mathrm{I} \otimes \mathrm{I})
$$

Corollary 4.4 Let us consider the contrast $\eta \rightarrow \infty$. Thus, the elastic inclusion degenerates to rigid one and the tensor $\mathbb{H}_{\infty}$ takes the form

$$
\mathbb{H}_{\infty}=-\frac{1}{4}\left(2 \frac{1+\beta}{\beta} \mathbb{I}-\frac{\alpha-\beta}{\alpha \beta} \mathrm{I} \otimes \mathrm{I}\right)
$$

Remark 4.5 From equality (78) we observe that the result given by theorem 4.2 represents the topological derivative of the Steklov-Poincaré operator (75). In addition, since solution $u \in K_{\omega}$ of the variational inequality (14)-(15) in $\Omega \subset \mathbb{R}^{2}$ is a $H^{1}\left(\Omega_{\gamma}\right)^{2}$ function, then it is convenient to compute the topological derivative from quantities evaluated on the boundary $\partial B_{R}$. In particular, we have the following representation for the strain tensor $\varepsilon\left(u\left(x_{0}\right)\right)$ [28]

$$
\begin{aligned}
\varepsilon_{11}+\varepsilon_{22} & =\frac{1}{\pi R^{3}} \int_{\partial B_{R}}\left(u_{1} x_{1}+u_{2} x_{2}\right) \\
\varepsilon_{11}-\varepsilon_{22} & =\frac{1}{\pi R^{3}} \int_{\partial B_{R}}\left((1-9 k)\left(u_{1} x_{1}-u_{2} x_{2}\right)+\frac{12 k}{R^{2}}\left(u_{1} x_{1}^{3}-u_{2} x_{2}^{3}\right)\right) \\
2 \varepsilon_{12} & =\frac{1}{\pi R^{3}} \int_{\partial B_{R}}\left((1+9 k)\left(u_{1} x_{2}+u_{2} x_{1}\right)-\frac{12 k}{R^{2}}\left(u_{1} x_{2}^{3}+u_{2} x_{1}^{3}\right)\right)
\end{aligned}
$$

where

$$
k=\frac{l^{*}+m}{l^{*}+3 m} .
$$

Once the above integrals are evaluated e.g. numerically, then we can use the constitutive relation (60) to compute the stress tensor $\sigma\left(u\left(x_{0}\right)\right)$. Finally, these results can by used to compute the topological derivative through formula (105).

\subsection{Approximation of solutions for variational inequalities}

We define a variational inequality for the crack problem with a perturbed bilinear form. The bilinear form is defined in the whole domain of integration, 
it is bounded and coercive on the energy space for the crack problem without any inclusion, and provides the first order topological sensitivity for the solutions of nonlinear elasticity boundary value problem with the nonlinear crack.

\section{Approximation of crack problem in $\Omega_{\delta}$}

We determine the modified bilinear form as a sum of two terms, as it is for the energy functional, the first term defines the elastic energy in the domain $\Omega$, the second term is a correction term, determined in Section 4.3. The correction term is quite complicated to evaluate, and we provide its explicit form, such a form is actually defined by the formulae in Section 4.3. The values of the symmetric bilinear form $a(\delta ; \cdot, \cdot)$ are given by the expression

$$
a(\delta ; v, v)=a(u, u)+\delta^{2} b(v, v) .
$$

The derivative $b(v, v)$ of the bilinear form $a(\delta ; v, v)$ with respect to $\delta^{2}$ at $\delta=0+$ is given by the expression

$$
b(v, v)=-2 \pi e_{v}(0)-\frac{2 \pi m}{l^{*}+3 m}\left(\sigma_{I I} \delta_{1}-\sigma_{12} \delta_{2}\right),
$$

where all the quantities are evaluated for the displacement field $v$ according to formulae in Section 4.3 where we provide the line integrals which defines all terms in (109), (110) and (111). Hence, we can determine the bilinear form $a(\delta ; v, w)$ for all $v, w$, from the equality

$$
2 a(\delta ; v, w)=a(\delta ; v+w, v+w)-a(\delta ; w, w)-a(\delta ; v, v) .
$$

In the same way the bilinear form $b(v, w)$ is determined from the formula for $b(v, v)$.

The convex set is defined in this case by

$$
K_{\delta}=\left\{v \in H_{\Gamma}^{1}\left(\Omega_{\delta}\right)^{2} \mid[v] \nu \geq 0 \quad \text { on } \gamma\right\} .
$$

Let us consider the following variational inequality which provides a sufficiently precise for our purposes approximation $u_{\delta}$ of the solution $u\left(\Omega_{\delta}\right)$ to crack problem defined in singularly perturbed domain $\Omega_{\delta}$,

$$
u_{\delta} \in K_{\delta}: \quad a(\delta ; u, v-u) \geq(F, v-u)_{\Omega_{\delta}} \quad \forall v \in K_{\delta} .
$$

The result obtained is the following, for simplicity we assume that the linear form $L(\delta ; \cdot)$ is independent of $\delta$.

Theorem 4.6 For $\delta$ sufficiently small we have the following expansion of the solution $u_{\delta}$ with respect to the parameter $\delta$ at $0+$,

$$
u_{\delta}=u(\Omega)+\delta^{2} q+o\left(\delta^{2}\right) \quad \text { in } H^{1}(\Omega)^{2},
$$


where the topological derivative $q$ of the solution $u(\Omega)$ to the crack problem is given by the unique solution of the following variational inequality

$$
\begin{array}{r}
q \in \mathcal{S}_{K}(u)=\left\{v \in\left(H_{\Gamma}^{1}\left(\Omega_{\gamma}\right)\right)^{2} \mid[v] \cdot \nu \geq 0 \quad \text { on } \Xi(u), \quad a(0 ; u, v)=0\right\} \\
a(q, v-q)+b(u, v-q) \geq 0 \quad \forall v \in \mathcal{S}_{K}(u)
\end{array}
$$

The coincidence set $\Xi(u)=\{x \in \gamma \mid[u(x)] \cdot \nu(x)=0\}$ is well defined [?] for any function $u \in H^{1}(\Omega)^{2}$, and $u \in K$ is the solution of variational inequality (114) for $\delta=0$.

For the proof of theorem we refer the reader to [27].

For the convenience of the reader we provide the explicite formulae for the terms in $b(v, v)$ defined by $(113)$, we refer to section 4.3 and to $[27,28]$ for details. We have

$$
\begin{array}{r}
2 \pi e_{\boldsymbol{v}}(0)=\frac{\pi\left(l^{*}+m\right)}{\pi^{2} R^{6}}\left(\int_{\Gamma_{R}}\left(v_{1} x_{1}+v_{2} x_{2}\right) d s\right)^{2} \\
+\frac{m}{\pi^{2} R^{6}}\left(\int_{\Gamma_{R}}\left((1-9 k)\left(v_{1} x_{1}-v_{2} x_{2}\right)+\frac{12 k}{R^{2}}\left(v_{1} x_{1}^{3}-v_{2} x_{2}^{3}\right)\right] d s\right)^{2} \\
+\frac{m}{\pi^{2} R^{6}}\left(\int_{\Gamma_{R}}\left[(1+9 k)\left(v_{1} x_{2}+v_{2} x_{1}\right)-\frac{12 k}{R^{2}}\left(v_{1} x_{2}^{3}+v_{2} x_{1}^{3}\right)\right] d s\right)^{2},
\end{array}
$$

with

$$
\begin{aligned}
& \sigma_{I I}=\frac{m}{\pi R^{3}} \int_{\Gamma_{R}}\left[(1-9 k)\left(v_{1} x_{1}-v_{2} x_{2}\right)+\frac{12 k}{R^{2}}\left(v_{1} x_{1}^{3}-v_{2} x_{2}^{3}\right)\right] d s, \\
& \sigma_{12}=\frac{m}{\pi R^{3}} \int_{\Gamma_{R}}\left[(1+9 k)\left(v_{1} x_{2}+v_{2} x_{1}\right)-\frac{12 k}{R^{2}}\left(v_{1} x_{2}^{3}+v_{2} x_{1}^{3}\right)\right] d s,
\end{aligned}
$$

and

$$
\begin{aligned}
& \delta_{1}=\frac{9 k}{\pi R^{3}} \int_{\Gamma_{R}}\left[\left(v_{1} x_{1}-v_{2} x_{2}\right)-\frac{4}{3 R^{2}}\left(v_{1} x_{1}^{3}-v_{2} x_{2}^{3}\right)\right] d s \\
& \delta_{2}=\frac{9 k}{\pi R^{3}} \int_{\Gamma_{R}}\left[\left(v_{1} x_{2}+v_{2} x_{1}\right)-\frac{4}{3 R^{2}}\left(v_{1} x_{2}^{3}+v_{2} x_{1}^{3}\right)\right] d s .
\end{aligned}
$$




\section{Acknowledgements}

This research is partially supported by the Brazilian-French research programme CAPES/COFECUB under grant 604/08 beetwen LNCC in Petropolis and IECN in Nancy and by the Brazilian agencies CNPq under grant 472182/2007-2 and FAPERJ under grant E-26/171.099/2006 (Rio de Janeiro). This work is completed during a visit of the first author to Nancy University whose support is appreciated very much. The work is also supported by Russian Fund for Basic Research (06-01-00209) and by the grant N51402132/3135 of the Polish Ministery of Education. These supports are gratefully acknowledged.

\section{References}

[1] S. Amstutz \& H. Andrä. A new algorithm for topology optimization using a level-set method. Journal of Computational Physics, 216(2):573-588, 2006.

[2] S. Amstutz, I. Horchani \& M. Masmoudi. Crack detection by the topological gradient method. Control and Cybernetics. 34(1):81-101, 2005.

[3] D. Auroux, M. Masmoudi \& L. Belaid. Image restoration and classification by topological asymptotic expansion. Variational Formulations in Mechanics: Theory and Applications - CIMNE, Barcelona, Spain 2007.

[4] M. Burger, B. Hackl \& W. Ring. Incorporating topological derivatives into level set methods. Journal of Computational Physics, 1(194):344-362, 2004.

[5] J.D. Eshelby. The Elastic Energy-Momentum Tensor. J. Elasticity. 5:321-335, 1975.

[6] G.R. Feijóo. A new method in inverse scattering based on the topological derivative. Inverse Problems, 20(6):1819-1840, 2004.

[7] G. Frémiot, W. Horn, A. Laurain, Murali Rao \& J. Sokolowski. On the analysis of boundary value problems in nonsmooth domains. Dissertationes Mathematicae, Institute of Mathematics of the Polish Academy of Sciences, in press.

[8] S. Garreau, Ph. Guillaume \& M. Masmoudi. The Topological Asymptotic for PDE Systems: The Elasticity Case, SIAM Journal on Control and Optimization, 39:1756-1778, 2001.

[9] S.M. Giusti, A.A. Novotny \& C. Padra. Topological sensitivity analysis of inclusion in two-dimensional linear elasticity. To appear on Engineering Analysis with Boundary Elements, 2008.

[10] M.E. Gurtin. An Introduction to Continuum Mechanics. Mathematics in Science and Engineering, vol. 158. Academic Press, 1981. 
[11] M.E. Gurtin. Configurational Forces as Basic Concept of Continuum Physics. Applied Mathematical Sciences, vol. 137. Springer-Verlag, 2000.

[12] I. Hlaváček, A.A. Novotny, J. Sokołowski and A. Żochowski. On topological derivatives for elastic solids with uncertain input data. To appear in JOTA, 2008 .

[13] M. Hintermüller. Fast level set based algorithms usind shape and topological sensitivity. Control and Cybernetics, 34(1):305-324, 2005.

[14] K.-H. Hoffmann, A.M. Khludnev, Fictitious domain method for the Signorini problem in linear elasticity, Adv. Math. Sci. Appl., 2004, vol. 14, N. 2, pp. 465-481.

[15] A.M. Khludnev, V.A. Kovtunenko, Analysis of Cracks in Solids, Southampton-Boston, WIT Press, 2000, 408p.

[16] A.M. Khludnev, Crack theory with possible contact between the crack faces, Russian survays in mechanics, 2005, vol. 3, N. 4, pp. 41-82.

[17] A.M. Khludnev, J. Sokolowski, Griffith formulae for elasticity systems with unilateral conditions in domains with cracks, Europ. J. Mech. A/Solids, 2000, vol. 19, pp. 105-119.

[18] A.M. Khludnev, J. Sokolowski, On differentation of energy functionals in the crack theory with possible contact between crack faces, J. Appl. Math. Mechs., 2000, vol. 64, N. 3, pp. 464-475.

[19] A.M. Khludnev, J. SokoŁowski, Smooth domain method for crack problem., Quarterly Appl. Math., 2004, vol. 62, N. 3, pp.401-422.

[20] I. Larrabide, R. A. Feijóo, A. A. Novotny and E. A. Taroco. Topological Derivative: A Tool for Image Processing. To appear on Computers \& Structures.

[21] T. Lewinski \& J. Sokolowski. Energy change due to the appearance of cavities in elastic solids. International Journal of Solids and Structures, 40:1765-1803, 2003.

[22] A. Morassi, E. Rosset, Detecting rigid inclusions, or cavaties, in an elastic body, Journal of Elasticity, 2003, vol. 73, pp. 101-126.

[23] S. A. Nazarov \& J. Sokolowski. Asymptotic analysis of shape functionals. Journal de Mathematiques Pures et Appliquees, 82(2):125-196, 2003.

[24] A.A. Novotny, R.A. Feijóo, C. Padra \& E. Taroco. Topological Sensitivity Analysis. Computer Methods in Applied Mechanics and Engineering, 192:803$829,2003$.

[25] A.A. Novotny, R.A. Feijóo, E. Taroco and C. Padra. Topological Sensitivity Analysis for Three-dimensional Linear Elasticity Problem. Computer Methods in Applied Mechanics and Engineering, 196:4354-4364, 2007.

[26] J. Sokolowski \& A. Żochowski. On the Topological Derivative in Shape Optimization. SIAM J. Control Optim. 37(4):1251-1272, 1999. 
[27] J. Sokolowski \& A. Żochowski. Modelling of topological derivatives for contact problems. Numerische Mathematik, 102:145-179, 2005.

[28] J. Sokolowski \& A. Zochowski. Topological derivatives for optimization of plane elasticity contact problems Engineering Analysis with Boundary Elements, 32(11): 900-908, 2008.

[29] J. Sokolowski \& J.-P. Zolésio. Introduction to Shape Optimization - Shape Sensitivity Analysis. Springer-Verlag, 1992. 